\title{
Die Dentinbildung in den Hautzähnen der Selachier.
}

\author{
Von
}

\author{
Dr. Carl Benda,
}

Assistent am pathol. Institut in Göttingen.

Hierza Tafel XVI.

Bereits Leydig, H. Meyer, Williamson und vor Allem Gegenbaur hatten klargelegt, dass die Hartgebilde der Selachierhaut vollkommene Homologa mit den Zähnen derselben Familie darstellen, und dass wir so in ihnen die Anfangsglieder in der Entwickelungsreihe der Wirbelthierzähne erblicken dürfen. Diese Beobachtung hat zuerst Oscar Hertwig ${ }^{1}$ ) in seiner Arbeit „Ueber Bau und Entwickelung der Placoidschuppen und Zähne der Selachier" verwerthet. Von der Erwartung, wie er in seiner Einleitung sagt, ausgehend, dass von den Selachiern, die auf einer niedrigeren Entwickelungsstufe stehen als Ganoiden und Teleostier, und die anatomische Verhältnisse zum Theil noch auf einer indifferenten Stufe der Entwickelung zeigen, auf so manche dunkle Punkte in der Zahnbildung der Säıgethiere einiges Licht geworfen werden könne, gelangt er zu einer wirklich erschöpfenden Würdigung der einzelnen, in die Zusammensetzung der gesammten Zahnanlage eingehenden Theile. Ferner liegt es seiner Arbeit, die Art und Weise, in welcher nun diese Theile die constituirenden Substanzen des fertigen Zahnes bilden, zu untersuchen, und so beribht er namentlich die Frage der Dentinbildung nur vorübergehend. Diese war nur von Leydig ${ }^{2}$ ) näher untersucht worden. Derselbe hatte an der Oberfläche der herausgenommenen Pulpa des Rochenstachels kugelige Kalkkörper beobachtet, die sich entweder noch isolirt, oder zu grösseren Klumpen verwachsen zeigen, Elemente, die er

1) Jenaische Zeitschrift. Bd. VIII, 1874. p. 331.

2) Beiträge zur mikroskopischen Anatomie etc. der Rochen und Haie p. 82. Lehrbuch der Histologie p. 94. 
den kurz vorher ron Czermak beschriebenen Zahnbeinkugeln an die Seite stellt. Er stellt sich das Wachsthum des Dentins so vor, dass der von den Pulpagefässen ausgeschiedene Kalk wohl in Vereinigung mit organischer Substanz zuerst kleinere Kugeln bildet, die sich durch Verschmelzung vergrössern und sich an die bereits gebildete Substanz anlegen; die Liicken zwischen den Kügelchen treten mit den schon fertigen verästelten Hohlräumen in Communication und bilden so die Kanälchen. Hertwig ubergeht diese Beobachtung gänzlich mit Stillschweigen und erwähnt nur, dass von ihm keine gegen die Ausscheidungstheorie sprechenden Beobachtungen gemacht wurden, wohl aber solche, welche, wie die Schichtungsstreifen im Dentin, sich mit der Umwandlungstheorie schwer vereinigen lassen. Wenn er sich auch ausdrücklich dagegen verwabrt, eine so wichtige Frage nur nebenbei zu entscheiden, so thut er eigentlich der Sache schon keinen guten Dienst, indem er die beiden Parteilosungsworte ,Umwandlungs - und Secretionstheorie" von vorn herein in die Untersuchung der Selachier hineinspielt, und so die Dunkelheit, die in diesem dunkelsten Punkte der Odontologie herrscht, ohne Weiteres dorthin uberträgt, wo sich nach seinem eigenen Ausspruche für andre Dinge so schönes Licht findet. Sollte man nicht vielmehr erwarten duirfen, auch in dieser Hinsicht bei den Hautzähnen der Selachier einfachere und ausgeprägtere Verhältnisse zu finden, und darum mit möglichster Unbefangenheit an die Untersuchnng der Vorgänge gehen? In diesem Sinne unternahm ich die beifolgende Arbeit über die Dentinbildung in den Hautzähnen der Selachier, und hoffe, sie so durchgeführt zu haben, wenn auch die Aussicht, gewonnene Resultate weiter ausdehnen zu dürfen, der Objectivität immer eine gewisse Gefahr bringt. Ich begann meine Untersuchungen im vorigen Herbst in Paris auf dem Laboratoire d'Histologie zoologique, wo mir G. Pouchet seinen Rath und sein reiches Material in liebenswürdigster Weise zur Verfïgung stellte. Herr Professor G. Fritseh hatte daun in Berlin die Freundlichkeit, sich für die Fortsetzung und den Abschluss der Arbeit zu interessiren. Beiden Herren sage ich hier meinen Dank.

Der Schwanzstachel von Trygon species (?), der mir als Beispiel der Rochenabtheilung vorlag, unterscheidet sich von dem allgemeinen Schema des Rochenstachels, wie es Le ydig beschreibt, dadurch, dass es bei ihm entsprechend seiner vorwiegenden Längs- 
ausdehnung nicht zur Bildung einer Centralhöhle kommt, von der die Gefässkanäle radiär ausstrahlen. Sie verlaufen vielmebr gleich von der Wurzel an ziemlich parallel und zeigen nur spïrliche Verästelung. Diese vollzieht sich vorkommenden Falls auf die gleiche Weise wie in den Haversischen Kanälen der Röhrenknochen, indem der Seitenast nach Bildung eines kurzen, auch schon möglichst spitzwinkligen Querverlaufes sofort die gleiche Richtung mit dem Hauptstamm annimmt. Ferner scheint der Stachel unterschiedlich von den kleineren nur an der vorderen und den Seitenflächen von einer Schmelzschicht iiberzogen zu sein. Die Kanälchen verlaufen, wie das Bild eines Querschliffs (Figur 1) zeigt, in einer breiten Zone unterhalb des Schmelzes, in der sich keine Gefässkanäle finden, ziemlich parallel untereinander senkrecht gegen den Schmelz. In den übrigen Partieen dagegen bieten sie das Bild einer grossen Unregelmässigkeit. Sie bilden ungefähr in der Mitte zwischen den Gefässkanälen ein dichtes Geflecht, aus dem sie sich plötzlich in einige grössere Kanälchen sammeln, die dann zu den Gefässkanälen hinziehen. In Folge dessen umgiebt jedes Gefäss ein ziemlich breiter Ring einer fast compacten Grundsubstanz, der sich namentlich bei schwacher Vergrösserung durch eine helle Farbe scharf gegen die grau schraffirten Mittelpartien abhebt. Eine sonstige lamellöse Schichtung der Grundsubstanz habe ich nicht wahrgenommen.

Querschnitte, die einem in Müller'scher Fltissigkeit conservirten Organe nach Entkalkung mit verdünnter Salzsäure entnommen waren, bieten ein wesentlich anderes Aussehen. Zwar imponirt der nur von wenigen scharf contourirten Kanälchen durchsetzte Ring in der Umgebung der Gefässe noch immer durch ein homogenes compactes Aussehen, in den iubrigen Theilen aber sind die feineren Kanälchen fast gänzlich verschwunden. Dagegen treten hier theilweise im Zusammenhang mit der compacten Substanz, theilweise unter einander verschmelzend, theilweise endlich ganz isolirt runde Scheiben auf, die sich circumscript gegen ein fein granulirtes Stroma, das sie einbettet, abheben. Da sie sich auf' Schnitten in allen Richtungen wiederfinden, nur dass sie auf Längsschnitten im Ganzen etwas elliptische Gestalt zeigen und oft auch zu Fasern aufgereiht sind, sind wir berechtigt, sie fuir den A tsdruck kugeliger Gebilde anzusprechen. In Bezug auf ihr Brechungsvermögen und namentlich durch ihr Verhalten gegen 
gewisse Farbstoffe schliessen sie sich der compacten Substanz an. Die compacte Substanz nämlich sowohl als die Kugeln resp. Fasern fărben sich in Ammoniakkarmin etwas dunkler als die granulirte Zwischensubstanz, und bleiben andrerseits durch in Wasser lösliches Anilinviolett vollkommen ungefärbt, wälrend die granulirte Substanz eine leichte violette Färbung annimmt.

Auf manchen Schnitten, und zwar um so häufiger, je mehr man sich der Wurzel des Organs nähert, zeigen sich in jener Region mitten zwischen den Gefässkanälen, die ich kurz als globuläre Substanz bezeichnen will, äusserst charakteristiscbe Luicken. Sie sind nämlich rings von den Kugeln begrenzt, die von allen Seiten ins Lumen hineinragen. Oft findet man auch Querschnitte derselben isolirt in der Lücke, die dann entweder wirklich frei in den Räumen liegen oder von einer über oder unter der Schnittfläche liegenden Wand hineinragen. Diese Lücken sind von sehr verschiedener Grösse. Manchmal scheint es, dass sich nur ein isolirtes Kanälchen in der granulirten Substanz erhalten hat, in andern fehlt einfach zwischen einigen Kugeln das Stroma, die grösseren schliesslich entsprechen einem Defect mehrerer Kugeln. Der Inhalt besteht aus einer feinkörnigen protoplasmaartigen Masse, die sich mit Anilinviolett intensiv färbt. Ich bezeichne diese Liicken wegen einer sofort imponirenden Aehnlichkeit als Interglobularräume. Es sind dies möglicher Weise dieselhen Räume, die Leydig in dem Basaltheil des Rochenstachels erwähnt, olne sie näher zu beschreiben. Ob sie aber dem entsprechen, was Hertwig in der Basalplatte der Placoidschuppe gesehen hat, ist mir sebr zweifelhaft. Derselbe nennt dort Interglobularräume gewisse ,kuglige Räume, die mit einer etwas vom Dentin verschiedenen Masse erfüllt sind". Nach meiner Auffassung ist aber ein Interglobularraum nichts weniger als kugelig, und sein Inhalt, ein protoplasmatischer Detritus unterscheidet sich auch recht erheblich vom Dentin. Sonst ist nur noch $\mathrm{zu}$ bemerken, dass eine besonders als Basaltheil differenzirte Partie nicht in dem Trygonstachel zu erkennen war, wenn auch sicher die Wurzelpartien sich durch eine reichlichere Bindegewebsverkalkung dem anschliessen. Soviel iiber das in seiner Entwicklung abgeschlossene Organ. Eins der mir vorliegenden bot in den Wurzelpartien Veränderungen des Baues dar, die zu dem Schlusse berechtigten, dass es noch im Wachsthum begriffen sei, und dieses benutzte ich zum Studium der 
Dentinbildung. Hier fanden sich nämlich die grossen Gefässe bis zu einer gewissen Höhe von einer kegelfö̀rmigen Pulpa umkleidet. Ein Querschnitt nahe der Wurzel zeigte demnach das Bild, das Fig. 2 darstellt. Die Grundsubstanz bildet in schmalen Balken ein netzartiges Gerïst von einer gewissen Regelmässigkeit, dessen Maschenräume ein oder mehrere centrale Gefïsse und die zellige Pulpa enthaltcn. Je weiter von der Wurzel die Querschnitte fallen, um so mehr verdicken sich die Balken der Grundsubstanz, um so geringer wird der Pulparaum, bis derselbe schliesslich auf einen einfachen Gefässkanal reducirt ist und wir so das Bild des ausgewachsenen Stachels haben.

Figur 3 stellt einen Querschnitt aus den untersten Theilen des Organs bei 500 facher Vergrösserung dar. Der Balken bereits fertigen Dentins (Fig. 3 G) zeigt denselben Bau, wie die globuläre Substanz des fertigen Stachels, der er ja nach seiner Lage in der Mitte zwischen den Gefässen auch entspricht. Wir seben in ihm das mattviolett gefärbte granulirte Stroma und die stark brechenden ungefärbten Kugeln. Gegen die Pulpahöhle zu zeigen die Balken eine höchst unregelmässige Grenze. Die Kugeln springen meistens weit in die Höhle vor und finden sich stellenweise ganz isolirt mitten unter den Zellen der Pulpa. Diese andrerseits dringen zum Theil so tief in die Balken ein, dass sie fast die Zellen der Nachbarhöhle erreichen, und sind an andern Stellen abgesprengt in den Balken ganz von Kugeln umgeben. Die Zellen der Pulpahöhle sind in derselben Weise, wie es Hertwig an andern Objecten beobachtete, nach zwei Richtungen hin differenzirt. In der Umgebung des centralen Gefässes finden sich auf einem ziemlich circumscripten Terrain, dessen Grenzen oft noch zum Ueberfluss durch lang ansgezogene Pigmentzellen bezeichnet sind, kleine Bindegewebszellen, deren kleiner runder Kern sich durch Anilinviolett etwas dunkler färbt, als das Protoplasma. Ausserhalb dieses Bezirks gewahrt man die unregelmässig abgeplatteten, im Ganzen rundlichen Querschnitte von Zellen, wie sie Hertwig und ich ganz typisch an all den Stellen wiederfanden, wo Dentification bei Selachiern vor sich ging, und die man daher füglich als Odontoblasten bezeichnen kann. Dieselben sind charakterisirt durch den grossen ovalen granulirten Kern, der nur von wenigem Protoplasma spindelförnig umgeben ist. Das Anilinviolett färbt, wie ich ganz regelmässig beobachtete, das Protoplasma intensiv und lässt den 
Kern ungefärbt. Diese Zellen liegen in der Pulpa des Trygonstachels ziemlich unregelmässig durcheinander, jedoch im Ganzen mit ihrer Längsrichtung derjenigen des Organs parallel. Endlich enthält jede Pulpahöhle namentlich gegen die Balken zu reichliche Mengen von krümligem Protoplasma, das man nicht in Zusammenhang mit cellulären Elementen sieht.

Ich will nun versuchen, wahrheitsgetreu den Weg zu berichten, den meine Beobachtungen und Schliisse in Betreff der Dentinbildung inne hielten; ich hoffe auf diese Weise am besten zu gleicher Zeit von meiner Gewissenhaftigkeit Zeugniss ablegen und den Vorgang demonstriren zu können. Ich untersuchte zuerst nur die tiefsten Theile anf Längs- und Querschnitten, indem ich erwartete, in den Gegenden, wo der Process vermuthlich am lebhaftesten war, die besten Bilder zu bekommen; ich färbte damals noch mit Eosin und hatte nur schwache Vergrösserungen bis $300 \mathrm{~d}$ zu meiner Verfügung. Das Eosin färbt alle protoplasmatischen Bestandtheile roth und lisst die dentificirten fast ungefärbt. Hierdurch heben sich diese Theile sehr scharf gegen einander ab, und namentlich die isolirten Kugeln im Innern der Pulpa treten deutlich hervor. Es sind dies offenbar dieselben Kalkconcretionen, die, wie oben erwähnt, schon Leydig sah, und die er für die eigentlichen Elemente des Dentins im Rochenstachel hält, indem er annimmt, dass sie sich an die schon gebildete Dentinsubstanz anlagern und diese so vermehren. Es kann in der That kaum zweifelhaft sein, dass diese Kugeln mit denen der globnlären Substanz und folglich überhaupt mit Theilen der Dentingrundsubstanz histologisch und genetisch zusammengehören. In diesem Falle beweist allein das Vorkommen jener isolirten Kngeln, dass die Grundsubstanz der Dentinbalken nicht das Secret einer epithelartigen Odontoblastenfläche sein kann, die man sonst allenfalls trotz ibrer Unregelmässigkeit noch zusammenconstruiren könnte. Vielmehr müssen auch dort die Bildungsvorgänge so beschaffen sein, dass in ihnen auch die Möglichkeit zur Entstehung derartiger isolirter Elemente gegeben ist. Prüfen wir also vorläufig einmal die verschiedenen Wege, die nach bekannten Analogien zur Bildung solcher einzelnen Kugeln führen könnten. Einige derselben lassen sich durch die Betrachtang des beschriebenen Bildes schnell auss chliessen. Erstens ist es undenkbar, dass eine einzelne der Spindelzellen, deren Querschnitte im Allgemeinen doch immer noch 
rund neben den runden Kugelquerschnitten liegen, wenn sie auch plötzlich noch so viel Auslïufer treibt, neben sich eine Kugel aussondern könnte. Jeder Anhalt fehlt ferner dafür, dass sich eine Gruppe dieser Zellen vereinigen sollte, um zwischen sich die Dentinkugel etwa in der Art auszusondern, wie die Epithelien eines Threoidfollikels eine homogene Colloidmasse.

Eine sehr naheliegende Analogie dagegen bietet die Bildung von Knochenkugeln, wie sie sich bei der unregelmässigen Ossification der Rhachitis oft im Innern einer Knorpelkapsel findet. Gleichviel, ob wir uns diese Bildung von einer Zelle, die auf Kosten der eigenen Substanz secernirt, oder von einer solchen, die eine successive Umwandlung ihrer äussersten Schichten erfährt, ausgehend denken, wir werden in diesem Falle immer eine Zelle oder den deutlichen Rest einer Zelle im Centrum der Kugel finden, und so werden wir auch nur aus diesem Befunde in unserm Falle ein gleiches Zustandekommen der Dentinkugeln annehmen dürfen.

Die Kugel könute sehr wohl auch im Innern der Zelle, wie der Fetttropfen in der Bindegewebszelle ausgesondert werden, in diesem Falle würde der Nachweis eines bei Seite gedrängten Kernes das Entscheidende sein. Sie könnte endlich nach Waldeyer's Dentificationstheorie aus der formellen und chemischen Umwandlung eines Theiles des Odontoblasten hervorgehen und auch in diesem Falle müsste sich das Wesen des Vorganges aus dem Verhalten der unveränderten Theile erkennen lassen.

Die genaueste Betrachtung meiner Objecte selbst mit Zuhülfenahme stärkerer Vergrösserungen entschied von diesen Punkten nur, dass sich niemals Reste einer wirklichen Zelle im Innern eines Globulus fanden, denn einige unregelmässige Risse, die hin und wieder woll hervortraten, waren absolut nicht dafür zu deuten. Aber auch die anderen Eventualitäten fanden nur Widerlegungen, denn nirgends verloren die Kerne von ihren äusserst präcisen Contouren etwas an Form oder Schärfe. Doch schien mir soviel nach diesem Bilde festzustehen, dass, gleichviel auf welche Weise, jedenfalls sehr plötzlich der Globulus an die Stelle des ganzen Odontoblasten treten musste. Von einer verkalkenden Intercellularsubstanz, die ich iibrigens nicht gesehen hatte, musste dann die granulirte Substanz, oder durch Liicken zwischen den Kugeln die Kanälchen gebildet werden; wo Zelle unmittelbar neben Zelle dentificirte, musste in späteren Phasen die compacte Substanz ent- 
stehen. Pouchet, der meine Präparate in Paris sah, schloss aus jenen Bildern auf eine von Zellen unahhängige Entstehung der Kugeln, wie er sie für die Hornfasern der Fischflossen, wie sie Ranvier für die elastischen Fasern annimmt.

War meine Auffassung die richtige, so musste ich das Genauere an solchen Stellen erkennen können, wo der Dentificationsprocess langsamer war, Stellen, wie sie sich doch bei der Entwickelung eines derartigen Organs finden mussten, und wirklich in exquisiter Weise fanden. Einmal war überhaupt anzunehmen, dass sich in weiter von der Wurzel entfernten Partien an den Spitzen der Pulpakegel die Vorgänge langsamer vollzogen. Darum untersuchte ich Schnitte aus diesen Gegenden. Diese Schnitte wiesen aber sofort noch eine andre, wohl noch bequemere Gelegenheit auf. Es fanden sich nämlich dort in Mitten der schon fast vollendeten Dentinbalken, die schon gegen die Pulpahöhle hin compacte Substanz zeigten, Interglobularräume, die den vorher beschriebenen vollständig glichen, nur dass ihr Inhalt meist deutlich zellig war. Die Entstehung dieser Interglobularräume war klar; bei der Unregelmässigkeit der ersten Dentificationsvorgänge hatte man schon an den andern Schnitten bemerken können, dass einzelne Zellen und Zellgruppen in das Innere der Dentinsubstanz abgesprengt wurden. Nun fanden sie sich aber als Interglobularräume an Stellen, wo sie später versehwanden; sie mussten also noch nachträglich der Limwandlung anheimfallen, und zwar in Folge der jedenfalls mehr erschwerten Stoffzufuhr vermuthlich äusserst langsan. Trotz alledem war selbst in diesen bequemen Objecten nichts zu finden, was meine Ansicht bestätigen konnte, oder mir sonst Klarheit verschaffte; itberall wo ich Zellen sah, fehlte auch nicht der scharf contourirte Kern, ja ich konnte sogar bemerken, dass das Protoplasma ihm gegenuiber immer mehr zuriicktrat, während andrerseits im Globulus nie Reste davon nachzuweisen waren, und dies waren ja die Punkte, auf die ich mein Augenmerls richtete. Ich suchte nun der Sache dadurch näher zu kommen, dass ich verschiedene Kernförbenittel anwandte. Hämatoxylin und Ammoniakkarmin (wie ich jetzt nachträglich noch hinzufügen kann), iibrigens auch Alaunkarmin, welches sonst wohl am sichersten die Kerne färbt, liessen gänzlich im Stich. So kam ich schliesslich zu dem im Wasser loslichen Jodmethylanilin, das mir die überraschendsten Resultate gab. 
Wie oben erwälnt, färbt das Anilinviolett das Protoplasma intensiv violett, lässt den Kern indess bei den Odontoblasten, unterschiedlich von dem der Bindegewebszellen ungefärbt. Hierdurch stellt sich in den Bildern eine eigenthümliche Beziehung zwischen ungefärbten Kernen und Kugeln, gefärbtem Protoplasma und granulirter Substanz her. Ich konnte jetzt wahrnehmen, dass einzelne der Interglobularräume Zellen enthielten, die vollstïndig den Character der Odontoblasten der Pulpa bewahrt hatten: intensiv gefärbtes Protoplasma und den grossen ovalen granulirten Kern. In andern konnte man den Kern anfinglich immer noch im Innern einer geformten Zelle, gewöhnlich mit betrüchtlicher Zunahme seines Volumens sein granulirtes Aussehen verlieren sehen. Dieses macht wohl meist zuerst in den Randpartien einer compacten Homogenitait Platz, denn öfters sieht man im Centrum noch ein granulirtes dunkleres Fleckchen, das ganz allmählich in die homogene Substanz aufgeht. Oft erhält sich auch die Granulirung in unregelmässigeren Linien. In einem weiteren Stadium verliert nun auch der Zellenleib seine Form. Das Protoplasma diffundirt als eine krümlige Masse und füllt im Verein mit dem der Nachbarzellen, die gewöhnlich gleichzeitig diese Veränderungen eingehen, den Interglobularraum gleichmässig ans. Oft sieht man tibrigens auch in demselben Interglobularraum eine Zelle noeh wohlerhalten zwischen andern aufgelösten liegen. Nach und nach erreicht der Kern vollständig das Aussehen der Dentinkugel und in zweiter Linie gewinnt das Protoplasma die Eigenschaften der granulirten Substanz, anscheinend oft viel später, nachdem der Kern seine Metamorphose durchgemacht hat. Kurzum: die Dentinkugel ist ein metamorphosirter Zellkern, die granulirte Substanz metamorphosirtes Zellprotoplasma.

Diese Beobachtung fand nun ihre vollständige Bestätigung in den Pulparäumen der betreffenden Gegend. Denn hier, wo ja die Bildung der compacten Substanz vor sich gehen musste, fanden sich, wie Figur 4 bei $P$ darstellt, die Pulpahöhlen fast ganz von etwas kleineren, aber dicht gedrängt liegenden Kernen ausgefuillt, die offenbar das Produkt einer fortgesetzten Wucherung und Zelltheilung der Pulpazellen sind. Hin und wieder nur haben sich dazwischen einige Reste von Protoplasma erhalten. Diese Kerne sind anch äusserst geeignet zum Studium der Metamorphose, indem man dort unmittelbar nebeneinander alle Bilder von der aus- 
geprägten Granulirung bis zur vollkommensten Homogenität findet. Es sind dies iibrigens auch offenbar die Stellen, auf die Leydig's Beobachtung am besten passt und an denen sie vermuthlich gemacht wurde. Hier sieht man Kugel an Kugel, stellenweise selbst ganze Klumpen derselben sicl den Dentinbalken anlagern.

Wenn ich jetzt wieder zu der Untersuchung der tiefen Querschnitte zurückkehrte, so wurde es mir nun leicht, dieselben zu verstehen. Nicht im Innern des Globulus war der Kern, sondern in der Umgebung des Globulus der Rest des Protoplasmas zu suchen. Hier zogen erstens die erwähnten Anbäufungen von krümeligem Protoplasma an der Grenze der Balken die Aufmerksamkeit auf sich. Dasselbe muss ron aufgelösten und verschmolzenen Zellleibern herrübren. Man erkennt auch, dass es oft in die granulirte Substanz iibergeht, oder dieselbe auch im Innern der Balken zwischen den Kugeln ersetzt. Recht selten ist allerdings noch der geformte Zellenleib um den Globulus erhalten. Hier duirfte man sicher zu der Annahme einer grösseren Plötzlichkeit des Vorganges seine Zuflucht nebmen, um das Fehlen dieser wichtigen Uebergangsform zu. entschuldigen. Indessen gelang es mir in einem rein mechanischen Momente eine noch weit befriedigendere Erklärung dafür za finden. Nicht selten fallen nämlich in den Präparaten Figuren wie die auf Fig. 3 bei * angedenteten auf, scharf umgrenzte Protoplasmahaufen, die sich in den äusseren Contouren den benachbarten Zellen anreihen, die aber statt einen Kern in sich einzuscbliessen, halbmondförmig eine Lücke umgeben. Ich halte dieselben für Zellenleiber, aus denen bei der Schnittführung der Kern herausgerissen warde, und dies vermuthlich, weil er eine festere Consisteuz als das Protoplasma erlangt hatte. Bei sorgfältigem Nachsuchen fand ich übrigens hin und wieder anch wirklich Globuli im Innern von geformtem Protoplasma, so dass ich also auch hier diesen Vorgang als constatirt betrachten.konnte.

In welcher Weise haben wir nun die Verschiedenheit zwischen den Produkten der Kern- und Protoplasmametamorphose aufzufassen? Vorliegende Erfahrungen kann ich nicht für oder gegen die Möglichkeit anführen, dass neben dem aus der Umwandlung des Kerns hervorgehenden Produkt, welches in diesem Falle jedenfalls als das wesentliche zu betrachten ist, noch ein chemisch davon verschiedenes von dem Protoplasma gebildet werde. Eine Entscheidung dieser Frage würde für die Kenntniss des Verhältnisses 
dieser beiden Zellbestandtheile $\mathrm{zu}$ einander einen werthvollen Beitrag geben. Vorläufig war indess nur zu constatiren, dass das Protoplasma bei der Umwandlung der Interglobularräume eine geringere Disposition fiir den Process oder eine geringere Attraction für die infiltrirenden Substanzen bewies. Diese Differenz scheint insofern von allgemeiner Gültigkeit und charakteristisch für die Odontoblasten des Trygonstachels, als sie sich auch, wie wir jetzt sehen werden, bei der Entstehung des Kanälchens ausspricht.

Die Beobachtung Leydig's, nach der durch die Liicken zwischen den Globulis die Kanälchen gehildet werden sollen, ist insofern von imponirender Richtigkeit, als es nirgends gelingen wird, ein Kanälchen in einen Globulus eintreten za sehen. Da aber die Lücken nach meiner Auffassung ursprünglich stets von Zellprotoplasma ausgefüllt sind, schliesse ich weiter, dass nur dieses sich an der Kanälchenbildung betheiligt. Nun konnte ich mich mit Leichtigkeit überzeugen, dass in der compacten Substanz, wo kein Protoplasma in granulirte Substanz übergeht, die Kanälchenbildung einfach dadurch zu Stande kommt, dass das Protoplasma, das hin und wieder zwischen den Kernhaufen der Matrix erhalten war, unverkalkt blieb. Es war mir aber selbst dort schon unmöglich zu entscheiden, ob dieses Protoplasma einen wesentlichen Bestandtheil der Kanälchen bildet, und sich darin erhält. Mit Anilinviolett liess es sich nicht mit Sicherheit nachweisen. Noch schwieriger liegt die Frage bei der globulären Substanz, wo die Kanälchen bei der Entkalkung fast vollstïndig verschwanden, wo sie aber jedenfalls in der granulirten Substanz liegen mussten. Die ganze Unregelmässigkeit ihrer Anordnung macht so sehr den Eindruck der Zufälligkeit, dass man sehr wohl an eine secundäre Entstehung denken könnte, derart, dass die granulirte Substanz, in der wohl anfänglich in Folge ihres eigenthïmlich langsamen Umwandlungsprocesses verbältnissmässig viel organische Substanz mit wenig Kalksalzen imprägnirt ist, einer nachträglichen Schrumpfung unterläge und in Spalten auseinander trete. Vielleicht könnten viele dieser kanälchenartigen Lücken gar erst bei der künstlichen Austrocknung des Organs entstehen. Wenn aber schliesslich die Kanälchenbildung darauf zurtickgeführt wurde, dass gar kein Protoplasma verkalkte, so muss man auch eine allmähliche Entwickelung dieses Phänomens voraussetzen und diese wirde naturgemiss darin bestehen, dass auch zuerst in der globu- 
lären Substanz geringere unverkalkt bleibende Protoplasmareste die Kanälchenbildung bedingen. Hierdurch ist die Kanälchenbildung gleichzeitig der normale Anfang der spätern, offenbar auf einer Entwickelungshemmung beruhenden Bildung von persistirenden Interglobularräumen, wie wir sie in der Wurzel des Organes gefunden haben. Wenn wir vorher nämlich die nachträgliche Metamorphose der bei der ersten Dentinbildung abgesprengten Zellhaufen als den gewöhnlichen Vorgang wahrgenommen hatten, wird in späteren Perioden mit dem Nachlass der nutritiven und formativen Vorgänge in dem Organe ein Zeitpunkt kommen, wo diese nicht mehr ausreichen, die Veränderung an jenen offenbar unter erschwerter Nahrungszufuhr stehenden Zellen zu bewirken. Hierbei sehen wir dann nach und nach immer grössere Reste von Protoplasma als Lacunen in der granulirten Substanz oder als erweiterte Kanälchen unverkalkt zurückbleiben, bis das Material auch für die Metamorphose der Kerne nicht mehr ausreicht und ganze Zellen in den Räumen zurückbleiben. In den meisten Fällen werden diese einem allmählichen Zerfall entgegengehen und dann den körnigen Protoplasmainhalt der Interglobularräume bilden, wobei nicht ausgeschlossen ist, dass sie sich auch längere Zeit intact erhalten.

Ich möchte nun die Resultate dieser längeren Betrachtung dahin zusammenfassen: Die Dentinbildung im Schwanzstachel des Trygon beruht auf einer Metamorphose von Zelltheilen, bei der sich Kern und Protoplasma verschieden verhalten. Aus der Umwandlung des Kerns geht die Dentinkugel hervor. Ursprünglich combinirt sich mit dieser ein von ihr verschiedenes Umwandlungsproduct des Protoplasmas, die granulirte Substanz. Indem allmählich der Process im Protoplasma nachlässt, kommt es zur Bildung von Kanälchen und später von Interglobularräumen. Hiervon ganz getrennt gehen in der Pulpá weitere Veränderungen vor, so dass die dentificirende Matrix schliesslich nur noch aus Kernen besteht; hierdurch entsteht die compacte Substanz. Das wesentliche in dem Vorgang der Dentinbildung des Trygonstachels ist also jedenfalls die Metamorphose der Odontoblastenkerne.

Wenn ich es wagen wollte, von dem Gesichtspunkte aus, von dem ich meine Arbeit unternommen hatte, aus meinen Untersuchungen über die Selachier allgemeinere Schlüsse zu ziehen, 
so galt es zunächst, zu constatiren, ob nicht etwa der Vorgang, den ich bei der Dentinbildung des Trygonstachel beobachten konnte, selbst in dieser Familie isolirt dastand. Hertwig hat, soviel ich es controliren konnte, von seinem Standpunkte aus mit Recht die Selachier als solidarisch aufgefasst. Für meine Frage musste schon der Unterschied zwischen Leydig's und Hertwig's Beobachtungen sehr verdächtig sein. An den ersteren Forscher konnte ich mich ohne Weiteres anschliessen; ein Blick auf Hertwig's Figuren aber musste genügen, zu zeigen, dass hier ganz andre oder sehr modificirte Vorgänge vorlagen.

Ich habe als Typus des Placoidbelages der Haifische den Flossenstachel vom Spinax acanthias untersucht. Der complicirte Bau dieses Organs ist äusserst interessant. Derselbe kommt durch eine Combination des Placoids mit Sceletttheilen zu Stande, welche sich mit einer Ausbreitung nicht des gewöhnlichen Hautplacoids, sondern einer Duplicatur desselben überdecken. Diese Bildung geht in der folgenden Weise vor sich. Wenn Hertwig bei den jtingsten Embryonen von Acanthias, die er untersuchte, solchen von $8 \mathrm{~cm}$ Länge, die Odontoblasten der zukïnftigen Placoidschuppen als langgestreckte Zellen mit grossen Kernen uber der bereits geschichteten Cutis an der untern Seite der Basalmembran angeschmiegt fand, so muss die Differencirung dieser Zellen von den Bindegewebszellen, die die geschichtete Cutis bilden, nattirlich wohl in der Zeit stattgefunden haben, da die Bildung der Cutis begann. Jedenfalls werden sich zu einer Zeit diese Zellen nicht von einander unterschieden haben, sondern einfach Rund- oder Spindelzellen des mittleren Keimblattes gewesen sein. Bei den etwas jüngeren Embryonen von etwa $6 \mathrm{~cm}$, die mir zur Verfugung standen, sah ich unter der Epidermis noch keine Odontoblasten und keine Cutis, sondern nur Spindelzellen, und eben diese Spindelzellen umbüllen die knorplige Anlage des vordersten Flossenstrahls der beiden Rückenflossen, die einen schief nach hinten und oben aufragenden Kegel darstellt. Einige Zwischenstufen fehlen mir, die ich ergänzen muss. Jedenfalls zu derselben Zeit, wo tiber der Bindegewebsbildung der Cutis einfache Zellen als Odontoblasten des Hautplacoids zurïckbleiben, wahrscheinlich an der Spitze mit diesen letzteren von vornherein zusammenhängend, differencirt sich aus den Spindelzellen uber dem Flossenstrahl eine kappenartige Schicht von Odontoblasten, während die unmittelbar 
an den Knorpel stossenden Zellenlagen in die Bildung gefässführenden Bindegewebes eintreten, das dazu bestimmt ist, gleichzeitig die Gefässpapille des Hautzahnes und das Perichondrium darzustellen. Dieses Bindegewebe schlägt sich an dem untern Rande der Odontoblastenschicht sattelförmig um, und hüllt so, indem auch die aussen gelegenen Spindelzellen Bindegewebe bilden, die Odontoblasten allseitig ein. In einem nächsten Stadium beginnt die Odontoblastenschicht zuerst an der Spitze einen sich sehr schnell uber die ganze Oberfläche des Flossenstrahls heruiberziehenden Dentinmantel zu bilden. Entsprechend der eigenthümlichen Lage der Odontoblasten zu ihrer Papille erfolgt diese Ablagerung aber nicht an der Oberfläche der Schicht, sondern in ihre Mitte, so dass die Schicht durch das von ihr gebildete Dentin in zwei Lamellen gespalten wird, die an dem untern Rande des Dentins in einem Ringe wieder sattelartig mit einander communiciren. Von beiden Lamellen, deren innere, dem Knorpel zunächst liegende die äussere bei weitem an Mächtigkeit übertrifft, geht jedenfalls das Dickenwachsthum der Dentinschicht aus, während der Sattel das Längenwachsthum versieht. Diese ganzen Vorgänge finden vorläufig noch unterhalb der unveränderten Hautdecke statt, die von vornher mit einem kleinen Höcker über den Stachel direct zur Flosse übergeht. Im weiteren Wrachsthum eilt nun der Stachel der Flosse voraus. Hierdurch hebt er die Haut, durchbohrt sie aber nicht eigentlich, sondern reisst sich mit der Hautdecke, die ihn bis zur Spitze an seinen Vorder- und den Seitenflächen überzieht, von der Flosse in der hinteren Region der äusseren Odontoblastenlamelle vor. Nun beginnt in diesem Hautzipfel, dessen Placoidodontoblasten, wie wir vorher erwähnten, mit denen des Stachels, jetzt also mit der äusseren Lamelle in Communication getreten sind, von der Spitze her die Placoidbildung. Die etwas schwierigen Verhältnisse dieses Placoids sind wohl so zu deuten, dass wir es uns als eine halbe kolossale Placoidschuppe des Hertwig'schen Schemas vorstellen, in deren Pulpa der Flossenstachel liegt. Auf diese Weise wird es klar, wie über den Stachel hin an seiner Vorderseite convergirende Gefässe zur Spitze aufsteigen, wie nm diese Spitze halbkreisförmig die vereinigten Odontoblasten des Placoids und der äusseren Lamelle liegen, und hier oben eine Substanz bilden, die der des Hertwig'schen Schuppenstachels entspricht, während die Substanz, die an den Seitenwänden gebildet wird, sich der der 
Basalplatte anschliesst. Wenn an der Spitze im Fortschritt des Wachsthums sich das Placoiddentin in immer grösserer Ausdehnung an das Dentin des Stachels anlegt, oblitterirt die zwischen ihnen befindliche Pulpahöhle bis auf die Gefässkanäle, die dann die Grenze zwischen den beiden Dentinarten noch im fertigen Stachel bezeichnen. Der unverkalkte Rest der Hautfalte, die das Placoid erzeugte, löst sich von diesem ab und legt sich von vornher an den Stachel, wie die Getreidegranne an das Fruchtkorn.

Wenn wir uns also den Bau des Organs noch einmal kurz vergegenwärtigen, so haben wir als Kern den schiefen Knorpelkegel des vordersten Flossenstrahls mit seinem Perichondrium. Dariiber stuilpen sich kappenartig die innere Odontoblastenlamelle und das Dentin, darauf die äussere Odontoblastenlamelle, die nach oben hin vorn bis auf einige Gefässkanäle oblitterirt, hinten dagegen zerrissen ist. Ein halber Kegelmantel von Placoid iiberdeckt das Ganze schliesslich nach oben und vorn.

Wir gehen jetzt auf den feineren Ban des Dentins ein, das uns hier wieder allein beschäftigen soll. Abgesehen von dem, nach dem Vorigen leicht verständlichen Zurïcktreten der Gefässkanäle in eine bestimmte Zone, tritt am besten auf Schnitten in der Nähe der Spitze, wo die Ausbildung des Dentins bereits vollendet ist, die Erscheinung bervor, die dem Dentin des Flossenstachels gegenüber dem des vorigen Organs am meisten ein charakteristisches Gepräge verleiht. Es ist dies die bedeutend hervorragendere Stellung, die die Kanälchen in dem ganzen Aufbau des Organes einnehmen. Dieselbe spricht sich vor Allem darin aus, dass ihr Inhalt jetzt deutlich protoplasmatisch ist; er färbt sich intensiv mit Anilinviolett und ist fein granulirt. Zweitens zeigt sich eine gewisse Regelmässigkeit in der Anordnung, in der sie die Grundsubstanz durchziehen. Sie dringen in diese von der Oberfläche aus in starken Stämmen ein, und verzweigen sich baumartig. Gemäss den drei Richtungen, in denen die Dentinbildung vor sich ging, bilden sie auf Querschnitten durch die obern Partien drei concentrische Systeme, nämlich eins im Placoid und zwei in dem andern Dentin. Die Richtung der Kanälchen ist im ganzen radiär, und zwar in dem Placoid im centrifugalen Sinne, in dem innern System des Stacheldentins ebenfalls centrifugal, im äussern centripetal. Die Kanälchen bezeichnen das Dentificationsgebiet jeder einzelnen Odóntoblastenschicht, und an der überwiegenden Mächtigkeit des 
innersten Systems erkennen wir, dass die innere Lamelle bei weitem den grössten Antheil an der Zahnbildung hatte. Die Zone, in der sich die letzten feinsten Ausläufer des von den beiden Lamellen ausgehenden Kanälchensystems auflösen, hat ein fein granulirtes Aussehen und färbt sich mit Anilinviolett diffus aber nur sehr schwach. Sie ist in den obern Theilen sehr schmal, nimmt aber nach unten zu an Mächtigkeit za, während die Kanälchen fübrenden Platten abnehmen. In den untersten Dentintbeilen sind keine Kanälehen. Auch die Lage des Dentins, die beidereits unmittelbar an die Odontoblasten stösst, hat ein granulirtes Aussehen. Man sieht die Kanälchen diese Schicht durchziehen, aber sie heben sich noch weniger von ihr ab. Das eigentliche Dentin setzt sich mit ziemlich scharfer, zackiger Grenze an diese granulirte Schicht an, während es in die andre allmählich übergeht.

Die Odontoblasten überziehen die Oberfläche des Dentins in einer ziemlich regelmässigen Sehicht im Vergleich mit denen des Trygonstachels. Sie verhalten sich genau wie die der Selachierzähne, wie sie Hertwig beschreibt. Von denen höherer Thierzäbne weichen sie allerdings noch erheblich, wie Hertwig bemerkt, darin $a b$, dass sie entsprechend der besondern Ursprungs- und Verlaufsweise der Dentinröhrchen zerstreut liegen, und nicht neben einander stehend zu einer Art von Cylinderepithel angeordnet sind. Es sind dieselben grosskernigen Spindelzellen nit spärlichem Protoplasma, wie sie bereits verschiedentlich erwähnt wurden, sie verhalten sich wie die andern gegen das Anilinviolett, doch gelang es mir, ihren Kern auch mit dem Grenacher'schen Alauncarmin zu färben. Ihre Richtung entspricht im Ganzen der Längsrichtung des Organs; auch in dem Sattel unter dem Dentin sind sie so geordnet und liegen hier also senkrecht gegen die Dentinschicht, der sie sonst platt anliegen. Der wesentlichste Unterschied dieser Zellen gegen die des Trygon ist, dass man sie häufig im Zusammenhang mit dem Protoplasmainhalt der Kanälchen sieht, so dass. es den Anschein hat, dass sie einen Fortsatz dort hineinsenden. Wir sehen also, dass sich das Bild hier erheblich dem aus den höheren Klassen nähert. In gleichem Schritte hiermit wächst nun allerdings auch die Zweideutigkeit der Dentificationsvorgänge. Die Frage spitzt sich hier thatsächlich ebenso zu, wie bei den höhern Thieren: Ist die Dentinbildung ein Vorgang, bei dem sich im Wesentlichen nur die oberste Odontoblastenschicht betheiligt, 
indem sie vor sich her das Dentin aussondert, oder wird dieses dadurch gebildet, dass die Odontoblasten Schicht auf Schicht dort eintreten, indem sie sich nach Waldeyer's Ausdruck chemisch und formell umwandeln. In diesem Falle war mir die wichtigste Frage: Ist es wieder, wie beim Trygon der Kern der Zelle, in dem sich der wesentliche Umwandlungsprocess abspielt? In dieser Hinsicht stellte ich von vornherein meine Untersuchungen an. Die ersten Resultate waren dem im höchsten Grade ungünstig. So aufmerksam ich die granulirte Substanz durchsuchte, ich konnte keine Spur von Kngeln oder Interglobularräumen dort finden. Sehr täuschende Bilder boten Querschnitte durch die untern Partien des Placoids dar, in denen sich reichlich runde, stark brechende Scheiben fanden. Dieselben stellten sich aber als Querschnitte mit die Verkalkung eintretender Bindegewebsblindel, die sich wie Sharpey'sche Fasern verhalten, heraus. Ebensowenig konnte ich wahrnehmen, dass das Dentin sich gegen die Odontoblastenlamelle kuglig abgrenzte.

Eine Stelle findet sich in dem Organe, die zum Studium der Erscheinungen von der Natur destinirt scheint, das ist diejenige, wo die Odontoblasten des Placoids und der äussern Lamelle zusammenstossen, und wo unter Neubildung von Dentin die Matrix um einige Gefässkanäle herum verschwindet. Diese bezeichnen dann noch die Grenzen, die jene ursprünglich einnahm. Es legen sich hier, wie das oben ausführlicher beschrieben wurde, zwei Dentinplatten an einander, deren Wachsthum, nach der Richtung ihrer Dentinröhrchen beurtheilt, fast in gerade entgegengesetzter Richtung vor sich gegangen war. Stellen wir uns nun vor, dass dieses Wachsthum von einer aussondernden Thätigkeit einer gewissen Anzahl von Odontoblasten abhängig gewesen war, und dass die entsprechenden Dentinröhrchen die Protoplasmafortsätze jener Zellen enthalten, die die dazu gehörige Intertubularsubstanz bildeten, so muss erstens an der Mündung der grösseren vielverzweigten Röhrchen ein ganzer Knäuel Odontoblasten zusammenliegen, in ähnlicher Weise, wie sie Hertwig in der Spitze der Schuppenpulpa am Ausgang des grossen centralen Dentinrohrs sah. Naturgemäss werden dieselben dann weiter, wenn sie von beiden Seiten aneinanderstossen, nach unten umbiegen und sich in dieser Richtung unter fortwährender Dentinausscheidung zurïckziehen, so dass hierdurch die Oblitteration der Pulpahöhle zu Stande käme. Viele 
der Odontoblasten würden sich auch secernirend in die Umgebung der Gefässe zurückziehen, und hier schliesslich eine wahre vascularisirte Pulpahöhle bilden. Dieses Alles wird sich schliesslich durch den Verlauf der Dentinröbrchen nachweisen lassen, wenn wir in einer Partie, in der die Höhle bereits vollständig verschwunden ist, die Stelle, die sie vorber einnahm, untersuchen. Wir finden hier also Stellen, an denen jede Spur von Zellen fehlt. Ein Theil der Röhrchen schneidet allerdings, wenn er die Grenze seines Dentinsystems erreicht hat, auf dem Querschnitt scharf ab, so dass man sich vorstellen kann, dass die Röhrehen sich nach unten umgeschlagen haben. Andre münden auch offen in die Gefässkanäle, so dass ihre Zellen in Beziehung zu den Gefässen getreten sein konnten. Ferner aber zeigt sich, dass die Röhrchen in jener Grenzregion ibberhaupt ganz allgemein ihre vorher so scharf ausgesprochene Richtung verlieren und sich in der mannigfachsten Weise unter einander verflechten. Hierbei findet man nicht selten ein äusserst bemerkenswerthes Bild. Ein Röhrchen wendet sich, bevor es die Grenzen seines Dentinsytems erreicht, in derselben Ebene mit einem sanften Bogen um, begegnet sich geradezu mit einem Nachbarröhrchen, und geht ohne jede Grenze in dieses ibber, so dass es den Anschein hat, als ob dasselbe Röhrchen in die Dentinsubstanz zurïckkehrt, und sich dort von Neuem verzweigt. In derselben Weise sieht man gar Röhrchen der beiden entgegengesetzten Dentinsysteme mit einander in Verbindung. Derartige Röhrchen sind sicher zufällig auf einander gestossen, die Odontoblasten aber,. die vorher vor ihrer Mündung lagerten, müssen, gleichviel welche Beziehung sie vorher zur Entstehung des Röhrchens hatten, hier an Ort und Stelle zu Grunde gegangen sein. Hier muss man beobachten können, ob sie atrophiren und resorbirt werden, oder ob sie in die Röhrchen oder die Grundsubstanz aufgehen.

Und in der That war es möglich, den Process zu verfolgen. Figur IX stellt einen Längsschnitt aus der betreffenden Gegend dar. Hertwig muss in seinen Placoidschuppen ganz ähnliche Bilder zu Gesicht bekommen haben. Er beschreibt in der Spitze der jungen Schuppe einen kegelförmigen Abschnitt der Pulpa, der in seiner Lage dem weiten Dentinrohr der ausgebildeten Schuppe entspricht, welches von der Papille direct nach der Spitze des Schuppenstachels dringt. Da er später in dem Rohre noch hin 
und wieder einige Odontoblasten vorfindet, und er von vorn herein der Ansicht ist, dass das Rohr zu Stande kommen muss, in dem sich die Odontoblasten secernirend daraus zuriickziehen, gelangt er weiter zu dem Schlusse, dass dieses Rohr sich von dem echten, nur Zahnfasern enthaltenden, dadurch unterscheidet, dass es eine Fortsetzung der Schuppenhöhle und sein Inhalt eine Verlängerung der Schuppenpulpa ist. Auch in unserm Bilde finden wir nach unten einen solchen Zipfel der Pulpa mit Odontoblasten, während von oben und seitwärts allerseits die Dentinröhrehen herantreten. Der Unterschied liegt allerdings darin, dass in unserm Falle aus dem Pulpazipfel kein weites Dentinrohr wird, sondern dass dieser Theil der Partie entspricht, in der sich jenes eigenthümliche oben beschriebene Flechtwerk von Dentinröhrchen bildet. Dies käme vielleicht dadurch zu Stande, dass die secernirenden Odontoblasten, die sich dort geordnet nach einer Richtung zurïckziehen, hier bunt durcheinander retirirten?

Wenn wir unsere Aufmerksamkeit zunächst den mittleren Theilen des Bildes zuwenden, muss die Aehnlichkeit derselben mit dem Interglobularraum des Trygonstachels in einem gewissen Stadium sofort in die Augen fallen. Wir erkennen eine Höhlung, die ebenfalls jene eigenthümlichen Begrenzungen zeigt, die wir als Abschnitte von Kugelcontouren ansprechen missen und ebenfalls liegen im Innern des Raumes kugelige Kalkconcretionen derselben Art, eingebettet in einem feinkrümeligen, protoplasmatischen Stroma. Mit völliger Sicherheit weisen alle möglichen Uebergangsformen darauf hin, dass die Analogie eine vollkommene ist, und wir jene Kugeln ebenfalls als die umgewandelten Kerne von Odontoblasten anzusehen haben, deren Protoplasma sich in den Lacunen zwischen den Kugeln erhalten hat. Dieses letztere verhält sich auch darin wie beim Trygon, dass es gleichzeitig mit der Dentification einer Auflösung unterliegt, und die zellige Begrenzung einbiisst, ein Vorgang, der hier zu einer weiteren wichtigen Erscheinung Anlass giebt. Die vorbereitenden Veränderungen treten in den Odontoblasten mit grösserer Regelmässigkeit als bei der Dentification in den Interglobularräumen auf, und so kommt es, dass die der Dentificationslinie zunächst liegenden Zellen wenigstens nach der einen Seite hin bereits ihre Grenzen verloren haben und unmittelbar mit dem Protoplasma, welches die Interglobularlacunen anfüllt, confluiren. Hierdurch gewinnt es den 
Anschein, als ob von ihnen aus Fortsätze in die Lacunen eindringen. Ein solcher Fortsatz ist eben nichts, als das zuriekgebliebene Protoplasma des vorigen Odontoblasten, welches mit dem der nächsten Zelle bereits bei Beginn der Kernmetamorphose in Continuität getreten war. Es muss hiernach einleuchten, dass niemals der Fortsatz eines Kerns in eine Lacune eindringen kann. Ein durchaus nicht seltenes und mit dem Wesen des Vorgangs wohl im Einklang stehendes Bild ist es dagegen, dass sich hin und wieder in den letzten Stadien des Processes ein nicht metamorphosirter Kern zwischen den Kugeln findet. Dies ist dann kein beim Riickzuge verspäteter Odontoblast, sondern dieselbe Entwicklungshemmung, die wir in den letzten Interglobularräumen des Trygonstachels ganz gewöhnlich antrafen.

Während soweit die Beziehungen des Interglobularraums zur Pulpa wohl vollkommen klargelegt sind, bleibt andrerseits sein Verhältniss zu dem andern Dentin zu untersuchen. Die Protoplasmalacunen gehen nirgend in eine der granulirten entsprechende Interglobularsubstanz über, sie scheinen also nicht zu einer nachträglichen Dentification bestimmt. Dagegen kann man sie nach allen Seiten bis tief in das anstossende Dentin hinein verfolgen. Sie dringen in dasselbe unter regelmässiger Verjüngung ihres Lumens und unter Absendung zahlreicher Verzweigungen ein, kurzum, die Protoplasmalacunen werden veritable Dentinröhrehen. Da nun umgekehrt die Röhrchen ohne eine merkliche Grenze in jenes mannigfaltig verzweigte Lacunensystem eindringen, wird es klar, wie es in jener Region zu der beschriebenen Verflechtung der Dentinröhrchen untereinander kommt und wie dann rein zufällig auf den Schnitten die Röhrchen jene oben beschriebenen eigenthümlichen Verbindungen und Anastomosen eingehen können, für die sonst jede Erklärung im Stich liesse.

Sollen wir nun annehmen, dass ebenso, wie nach Hiertwig's Ansicht das weite Dentinrohr, so hier der Interglobularraum eine ganz eigenthümliche Bildung ist, die wir von der iibrigen Dentification abtrennen milissen; sollen wir annehmen, dass nur jene letzten an der Mündung der Dentinröhrchen liegenden Odontoblasten eine solche Metamorphose eingehen könnten? Ich glaube vielmehr, dass wir uns insofern auf eine Homologie der Erscheinungen verlassen dürfeu, um in der Bildung jener Interglobularräume die Consequenzen derselben Vorgänge zu suchen, die bei der Entwickelung 
des ganzen Organes galten. Nach der Analogie des Trygonstachels werden wir erwarten dürfen, dass diese Vorgänge in einer grundlegenden Dentification der Odontoblastenkerne bestehen, neben welchen eine allmählich nachlassende Metamorphose des Protoplasmas einhergeht. Und in der That beweisen Bilder, wie sie Fig. VIII und $\mathrm{X}$ darstellen, hinlänglich, dass in den Anfangsstadien der Entwickelung ganze Zellen, und im weitern Verlauf stets noch Theile der Odontoblasten selbst in die Dentinsubstanz übertreten, und Zellreste die Dentinröhrchen ausfüllen. Es kommt aber nirgend zur Bildung von globulärer Substanz, sondern die Producte der Kern- und Protoplasmametamorphorse sind gleich. Dieser Umstand erschwert allerdings in hohem Grade gegeniiber dem Verhalten beim Trygon die Entscheidung der Frage, wie weit sich die einzelnen Zellbestandtheile bei der Dentinbildung betheiligen. Durch directe Anschauung kann man sieh nur liberzeugen, dass factisch nie ein Fortsatz des Kerns in ein Dentinrohr eindringt, sondern dass der Kern bis zu dem Moment, wo er in die Dentinmasse aufgeht, seine scharfen runden Contouren behält. Das Beweisendste bleibt nach meiner Meinung doch der Ausgang des Processes, wie wir ihn in dem Interglobularraum beobachteten, und ich trage daher kein Bedenken, auch in dem Flossenstachel des Dornhais die Bildung der Dentingrundsubstanz im Wesentlichen auf eine Metamorphose der Odontoblastenkerne zurückzuführen, neben der diejenige des Protoplasma nur von untergeordneter Bedeutung ist. Hier spricht sich die geringere Attraction des Protoplasma eben nicht in der Bildung einer anders gearteten Substanz, sondern nur dadurch aus, dass dasselbe zwar in der ersten Lebhaftigkeit des Processes dazu gelangt, eine dem andern Dentin gleiche Substanz zu bilden, dann aber sogleich ein allmähliches Nachlassen der Veränderungen die Röhrchenbildung einleitet. Eine gewisse Verschiedenheit des Protoplasmadentins documentirt sich ja auch in dem granulirten Aussehen der Zone, in der es in grösserer Mächtigkeit auftritt.

Wenn wir nun auch in dem allmählichen Nachlassen der Protoplasmametamorphose den Grund für das continuirliche $\mathrm{Zu}$ nehmen des Lumens der Röhrchen erblicken dürfen, so reicht dies noch nicht aus, um eine unverkennbare Regelmässigkeit des Verlaufes der Röhrchen zu erklären, der ja namentlich in den grösseren oft ganze Strecken weit vollkommen geradlinig ist. Woher 
kommt es z. B. in der Pulpaspitze der Placoidschuppe zur Bildung des graden weiten Dentinrohrs, während an ähnlicher Stelle in dem Flossenstachel ein unregelmässiger Interglobularraum entsteht? In letzterem Falle macht es vollkommen den Eindruck, als ob die interglobulare Lacune in ganz zufälligen Combinationen mit dem sich auflösenden Protoplasma der benachbarten Odontoblasten in Verbindung tritt. Zur Bildung eines einigermaassen regelmässigen Rohres ist aber entschieden nöthig, dass sich die dentificirenden Kerne vorher richten und dass sich in Folge dessen stets entsprechende Protoplasmaabschnitte an das Dentinrohr anschliessen. Finden sich nun Andeutungen eines solchen Vorganges?

In der Spitze der Schuppenpulpa beschreibt Hertwig eine Gruppe von Zellen, die ein besonderes Verhalten zeigen. Sie sind mehr cylindrisch, besitzen einen grossen Kern, und an ihrem peripheren Ende einen langen Ausläufer. Sie liegen, ohne eine distincte Schicht auf der Pulpa zu bilden, um die Einmündungsstelle des centralen Dentinrohrs, und schicken ihre Ausläufer zu einem Bündel vereint in dasselbe. Er unterscheidet sie von den platten Zellen, die an den Seitenwänden der Höhle langgestreckt dem Dentinrand anliegen, und nur kleine oder gar keine Ausläufer in das Dentin schicken. Ich habe ein derartiges Verhalten an der Stelle, wo es Hertwig beschreibt, vollkommen constatiren können, und habe es auch bei der Dentinbildung des Flossenstachels gewissermassen auf die Fläche projicirt wiedergefunden. Auch hier nämlich kann man anf gewissen Schnitten zwischen den platt anliegenden Odontoblasten ein ganzes Bündel von Zellen wahrnehmen, die im Ganzen senkrecht gegen das Dentin gerichtet vor einem Böhrchen liegen, dessen Protoplasmainhalt in Zusammenhang mit dem Zellenconvolut steht. Nur muss jch bemerken, dass diese Gruppe sich oft soweit von den Nachbarzellen absondert, dass sich in ihrem Innern kaum Zellgrenzen erkennen lassen und dagegen die ganze Gruppe fast wie eine Riesenzelle (Fig. VIII bei ${ }^{*}$ ) neben den andern imponirt. Hertwig, der sie entsprechend seinen Ansichten über die Dentinentwicklung für die Odontoblasten, die sich aus den einzelnen Verzweigungen des Rohrs zurückgezogen baben, hält, vermuthet, dass sie durch das bereits vollendete Rohr die Ernährung der fertigen Substanz übernehmen, während die platten Zellen in der Secretion fortfahren. Ich bin in Betreff dieser Zellgruppen zu einer völlig andern Ansicht gekommen. Es ist sehr 
sehwer, an einer Placoidschuppe zu entscheiden, ob sie sich noch in Stadium des Wachsthums oder der Vollendung befindet. In dem Stacheldentin aber konnte ich mit voller Sicherheit constatiren, dass jene Zellenhaufen nicht vor vollendeten Dentinröhren liegen. Sie finden sich vielmehr nur in den untern Partien des Stachels vor ziemlich kleinen Röhrchen und zwar fast ausschliesslich in der inneren Pulpalamelle, von der ja hauptsächlich das Dickenwachsthum des Dentins und die Bildung der grössten Dentinröhrchen ausging, also nicht an Stellen, wo grössere Deutinröhrchen entstanden sind, sondern dort, wo solche entstehen sollen. Ich habe mich somit berechtigt geglaubt, sie mit der Bildung dieser Röbrchen in Beziehung zu bringen, und halte sie für solche Zellen, die sich zum Zwecke der Röhrehenbildung rangirt haben. Man wird dieses Phänomen, wenn man nicht an Force majeure oder Instinct der Zellen glauben will, am natürlichsten aut das Vorhandensein eines gemeinschaftlichen Wucherungscentrums für jeden derartigen Zellencomplex zurïckzuführen haben. Hierdurch wird es leichter verständlich, dass die Zellen eines solchen Wucherungsherdes, da sie ja von vornherein schon einen innern Zusammenhang haben, nachher in einer gewissen Gruppirung in die Dentification eintreten, und einen regelmässig verlaufenden Röhrchenstamm bilden. Sie sind also durchaus nicht von den andern Odontoblasten specifisch verschieden. Vielmehr ist es sogar wahrscheinlich, dass sie, wenn sie gegen die Dentificationslinie vorgeschoben werden, sich platt gegen diese anlegen. Es ist auch nicht einmal zu beweisen, dass nicht jeder der platten Odontoblasten in einer andern als der Schnittebene mit einem derartigen Herde in Verbindung steht, oder nicht wenigstens doch einem solchen entstammt. Dennoch scheint allein in dem Umstande, dass eine grosse Zahl von Odontoblasten, die entweder unregelmässiger entstehen, oder sich vorzeitig von jenen Herden separiren, als isolirte Individuen dentificiren, jene Wucherungsherde dagegen vereinzelt auftreten, der Grund für das spärlichere Auftreten regelmässigerer und grösserer Röhrchenstämme zu liegen.

Ich trage kein Bedenken, die am Flossenstachel des Dornhais gewonnenen Resultate über die Dentinbildung vorläufig ohne Weiteres auf die von Hertwig untersuchten Selachierorgane, nämlich die Placoidschuppen und die Kieferzähne auszudehnen. Hier muss ich noch einmal Hertwig's Einwand gegen die Metamorphose der 
Odontoblasten berühren. Nach meiner Meinung beweisen Schichtungsstreifen, die ich zwar nicht bei den Stacheln von Trygon und Acanthias, aber wohl in den Zähnen gesehen habe, weiter nichts als eine Periodicität der Wachsthumsvorgänge, wie sie nach analogen Erscheinungen bei der Schalenbildung der Wirbellosen, bei Häutung und Mauserung von Epidermoidalbildungen u. s. w. sehr wohl denkbar ist. Dass aber beispielsweise die Bildung von Jahresringen als Beweis gegen die zellige Natur der Holzgefässe angeführt worden wäre, ist mir nicht bekannt.

Bei der Beobachtung von zwei Formen von Dentinbildung in der Selachierfamilie habe ich folgendes Resultat erhalten:

Die Grundlage der Dentinbildung liegt in der Metamorphose der Odontoblastenkerne. Das gleichzeitige Verhalten des Odontoblastenprotoplasma und die vorbereitenden Vorgänge in der Matrix bedingen Differencirungen in der Dentinform und dem Bau des Organes.

Gegenstand meiner nächsten Arbeiten wird der Nachweis sein, dass sich die Giltigkeit dieser Sätze über die engen Grenzen der Selachierfamilie ausdehnt. Der zweite Satz wird den Gesichtspunct für die Construction von Entwicklungsreihen abgeben, und gewisse Differenzirungen des Processes im Placoid gegenüber dem Rochendentin deuteten bereits den Weg an, auf dem sich diese Reihen zu höheren Formen bewegen werden.

Halle a. d. S., 11. Juni 1881.

\section{Erklärung der Tafel XVI.}

Fig. 1-5. Schwanzstachel von Trygon.

Fig. 1. Querschliff durch den ausgewachsenen Stachel. Vergrösserung 45 .

Fig. 2. Querschnitt durch den wachsenden Stachel $0,5 \mathrm{~cm}$ über dem Wurzelende. Vergr. 45.

Fig. 3. Dasselbe. Vergr. 500.

Fig. 4. Querschnitt durch den wachsenden Stachel $3 \mathrm{~cm}$ über dem Wurzelende. Vergr. 500.

Fig. 5. Längsschnitt durch die Wurzel des wachsenden Stachels. S Schmelzschicht, G Grundsubstanz des Dentins, P Pulpahöhle, V Gefäss, I Interglobularraum, a Zahnkugel, c granulirte Substanz, b Odontoblast, * kernlose Odontoblasten. 
Fig. 6-10. Flossenstachel von Acanthias.

Fig. 6. Längsschnitt, Uebersichtsbild. S Stachel, F Flosse, C Cutis, in einen Zipfel $G$ auslaufend, K Knorpel des Sceletts.

Fig. 7. Querschnitt durch die Spitze des Stachels. Vergr. ca. 30.

A Dentin der innern Pulpalamelle, B Dentin der äussern Lamelle, Pl. Placoiddentin, V Gefässe, G granulirte Schicht.

Fig. 8. Dentification der innern Pulpalamelle im Querschnitt. Vergr. 500. K Knorpel, Pc Perichondrium, $\mathbf{P}$ innere Pulpalamelle, A Dentin, b Odontoblasten, * Odontoblastenbündel, $\mathrm{k}$ Dentinröhrchen.

Fig. 9. Dentification im obersten Theil der äusseren Pulpalamelle. Längsschnitt. Vergr. 500. Pl. Placoid, P Pulpa, B Dentin, b Odontoblasten, h Dentinröhrchen.

Fig. 10. Dentification an der Umschlagsstelle der Pulpa am untern Dentinrand. Längsschnitt. Vergr. 500. P Pulpa, D Dentin, b Odontoblasten, ch Pigmentzellen. 